\title{
Proteomic and Metabolomic Characterization of Metabolically Healthy Obesity: A Descriptive Study from a Swedish Cohort
}

\author{
J. Korduner $\mathbb{D}^{1},{ }^{1,2}$ P. M. Nilsson $\mathbb{D}^{1,},{ }^{1,2}$ O. Melander $\mathbb{D}^{1},{ }^{1}$ M. J. Gerl $\mathbb{D}{ }^{3}{ }^{3}$ G. Engström $\mathbb{D}^{1}{ }^{1}$ \\ E. Bachus $\mathbb{D},{ }^{1}$ M. Magnusson $\mathbb{D},{ }^{1,4,5,6}$ and F. Ottosson $\mathbb{D}^{1}$ \\ ${ }^{1}$ Department of Clinical Sciences, Lund University, Malmö, Sweden \\ ${ }^{2}$ Department of Internal Medicine, Skåne University Hospital, Malmö, Sweden \\ ${ }^{3}$ Lipotype GmbH, Dresden, Germany \\ ${ }^{4}$ Wallenberg Center for Molecular Medicine, Lund University, Lund, Sweden \\ ${ }^{5}$ Department of Cardiology, Skåne University Hospital, Malmö, Sweden \\ ${ }^{6}$ North-West University, Hypertension in Africa Research Team (HART), Potchefstroom, South Africa
}

Correspondence should be addressed to J. Korduner; johankorduner@gmail.com

Received 16 December 2020; Accepted 23 September 2021; Published 6 October 2021

Academic Editor: Gordon Fisher

Copyright (c) 2021 J. Korduner et al. This is an open access article distributed under the Creative Commons Attribution License, which permits unrestricted use, distribution, and reproduction in any medium, provided the original work is properly cited.

\begin{abstract}
Background/Aims. Obesity is a well-established risk factor for the development of numerous chronic diseases. However, there is a small proportion of obese individuals that seem to escape these aforementioned conditions-Metabolically Healthy Obesity (MHO). Our aim was to do a metabolic and biomarker profiling of MHO individuals. Method. Associations between different biomarkers (proteomics, lipidomics, and metabolomics) coupled to either MHO or metabolically unhealthy obese (MUO) individuals were analyzed through principal component analysis (PCA). Subjects were identified from a subsample of 416 obese individuals, selected from the Malmö Diet and Cancer study-Cardiovascular arm (MDCS-CV, $n=3,443$ ). They were further divided into MHO $(n=143)$ and MUO $(n=273)$ defined by a history of hospitalization, or not, at baseline inclusion, and nonobese subjects (NOC, $n=3,027$ ). Two distinctive principle components (PL2, PP5) were discovered with a significant difference and thus further investigated through their main loadings. Results. MHO individuals had a more metabolically favorable lipid and glucose profile than MUO subjects, that is, lower levels of traditional blood glucose and triglycerides, as well as a trend of lower metabolically unfavorable lipid biomarkers. PL2 (lipidomics, $p=0.02$ ) showed stronger associations of triacylglycerides with MUO, whereas phospholipids correlated with MHO. PP5 (proteomics, $p=0.01$ ) included interleukin-1 receptor antagonist (IL$1 \mathrm{ra}$ ) and leptin with positive relations to MUO and galanin that correlated positively to MHO. The group differences in metabolite profiles were to a large extent explained by factors included in the metabolic syndrome. Conclusion. Compared to MUO individuals, corresponding MHO individuals present with a more favorable lipid metabolic profile, accompanied by a downregulation of potentially harmful proteomic biomarkers. This unique and extensive biomarker profiling presents novel data on potentially differentiating traits between these two obese phenotypes.
\end{abstract}

\section{Introduction}

Although obesity is a well-established risk factor for the development of endemic modern Western public health problems, including cardiovascular disease (CVD) and type 2 diabetes (DM2) [1], accumulating evidence is suggesting that there is a small proportion of individuals with excess weight (body mass index $(\mathrm{BMI}) \geq 30 \mathrm{~kg} / \mathrm{m}^{2}$ ) that seem to escape these aforementioned conditions-a concept known as Metabolically Healthy Obesity (MHO) [2, 3]. Along with this phenomenon, there has been a debate concerning the heterogeneity of obesity, and the negative consequences of excess fat seem to be more complex and individually patterned than previously thought $[2,4]$. However, there is no doubt that obesity in the majority of cases represents a state of increased risk. Even in obese individuals defined as MHO that seem to experience less negative effect of their excess weight, increasing evidence is suggesting that this could be a 
transient state and will eventually in due time transform into its unhealthier counterparts-Metabolically Unhealthy Obesity (MUO) [5-7].

There exists no agreed definition of $\mathrm{MHO}$, but most studies on this topic suggest that it should involve a lack of risk factors for the metabolic syndrome (MetS) [8]. In a recent paper [9], we defined $\mathrm{MHO}$ as obese individuals $\left(\mathrm{BMI} \geq 30 \mathrm{~kg} / \mathrm{m}^{2}\right)$ who had never been hospitalized for a somatic disease before study baseline (at mean age of 56 years) and described the prognosis regarding incident $\mathrm{CVD}$ and mortality risk of $\mathrm{MHO}$ subjects compared to MUO and nonobese controls (NOC) in a Swedish cohort from the 1990s-the Malmö Diet and Cancer Study (MDCS; $n=28,098$ ). Our findings suggested that MHO individuals had a significantly lower risk of total mortality and developing CVD during a 20-year follow-up period, compared to MUO individuals. Interestingly, no differences in prospective risks could be seen when comparing $\mathrm{MHO}$ to NOC individuals. Descriptive data from the study showed that $\mathrm{MHO}$ individuals presented with a less sedentary lifestyle, held a higher educational level, and displayed a more favorable glucose and lipid blood profile [9]. These descriptive findings were in line with earlier publications $[10,11]$, although our definition of $\mathrm{MHO}$ was novel and differed from previous ones [9].

There is still no clear explanation as to which factors contribute to the development of MHO contra MUO, but many theories exist. One common assumption is that a chronic inflammatory state, commonly associated with obesity, is downregulated in MHO individuals [12]. This in turn could be interpreted as influenced by less pronounced nonalcoholic fatty liver disease (NAFLD), determined by genetic factors and/or a diversity of the gut microbiota [4]. Other benign attributes that attract one's attention is the distribution patterns (peripheral vs. central obesity) [13] and the expandability of adipose tissue [14], as well as the glucose and triglyceride index $[15,16]$, but also specific biomarkers associated with obesity such as adiponectin [17] and neurotensin [18].

With this in mind, there is an urge to better understand the true benign nature of obesity presented in some selected individuals and the factors associated with it, to improve and individualize the treatment and care of individuals with excess body weight. From our recent paper [9], we concluded that a sedentary lifestyle and higher levels of blood glucose and lipids, combined with adverse lower socioeconomic conditions, contribute negatively to unhealthy obesity. Nonetheless, we would like to elucidate further on the descriptive profile of MHO individuals and thus analyze selected biomarkers associated with this specific phenotype.

Consequently, this observational study aimed to better characterize the metabolic profile of previously defined MHO individuals [9] by comparing plasma levels of metabolites (metabolomics and lipidomics) and circulating proteins (proteomics) between MHO, MUO, and NOC subgroups.

\section{Materials and Methods}

2.1. Subjects. A total of 28,098 individuals were selected (41\% attendance rate) to participate in the baseline examination of the MDCS between 1991 and 1996, which included risk factor assessment through laboratory testing, physical examination, and a questionnaire. A detailed description of the inclusion criteria [9] and methodological aspects has been previously published [19,20]. In short, obese individuals $\left(\mathrm{BMI} \geq 30 \mathrm{~kg} / \mathrm{m}^{2}\right.$ ) were selected from the subcohort MDCS-Cardio Vascular arm (MDCS-CV). This subcohort was derived from the original MDCS, when every other individual included in the baseline examination was reinvited during 1992-1994 to participate in MDCS-CV $(n=6,103)$. The primary aim was to study the epidemiology of carotid artery disease, when also laboratory analyses of additional fasting blood samples were carried out [21, 22].

The number of included individuals was further reduced due to the lack of complete biomarker profiling data $(n=3,443)$. The obese individuals were then subdivided into two groups consisting of $\mathrm{MHO}$ and MUO, based upon the absence of hospitalization for somatic disease up until the inclusion at MDCS-baseline examinations (MHO) [9]. Hospitalization status was obtained through the Swedish National Hospital Inpatient Register, where external injuries/intoxications and normal deliveries were considered nonhospitalization and excluded. Furthermore, hospitalization status included only records for somatic disease. Obese individuals with no recorded history of hospitalization were considered MHO $(n=143)$, whereas MUO individuals were characterized as individuals with at least one record of hospitalization for somatic disease prior to inclusion at MDCS-baseline $(n=273)$. Moreover, selected $\mathrm{MHO}$ individuals were further compared with NOC subjects from the same subcohort $(n=3,027)$; see Figure 1 for a detailed flowchart. This novel approach of defining $\mathrm{MHO}$ individuals has been previously applied in the same cohort from an urban population [9].

2.2. Metabolite Profiling. Profiling of plasma metabolites was performed using a LC-QTOF-MS System (Agilent Technologies 1290 LC, 6550 MS, Santa Clara, CA, USA) and has previously been described in detail [23]. Briefly, overnight fasted citrate venous plasma samples stored at $-80^{\circ} \mathrm{C}$ were thawed and extracted by addition of $120 \mu \mathrm{l}$ extraction solution ( $80: 20$ methanol/water) to $20 \mu \mathrm{l}$ plasma. The samples were then incubated at $4^{\circ} \mathrm{C}$ for 1 hour at $1250 \mathrm{rpm}$. After $15 \mathrm{~min}$ centrifugation at $14000 \mathrm{~g}, 100 \mu \mathrm{l}$ supernatant was transferred into a glass vial for analysis. Extracted samples were separated on an Acquity UPLC BEH Amide column $(1.7 \mu \mathrm{m}, 2.1 * 100 \mathrm{~mm}$; Waters Corporation, Milford, MA, USA). Metabolite identification, quality control, and normalization were performed as described previously [24].

2.3. Lipid Profiling. Lipid extraction of $1 \mu \mathrm{L}$ of overnight fasted citrate plasma samples was stored at $-80^{\circ} \mathrm{C}$ upon collection, followed by quantitative mass spectrometrybased lipid analysis. The analysis was performed at Lipotype $\mathrm{GmbH}$ using a high-throughput shotgun lipidomics technology [25]. Lipid identifiers of the SwissLipids database [26] (https://www.swisslipids.org) are provided in Table S1. 


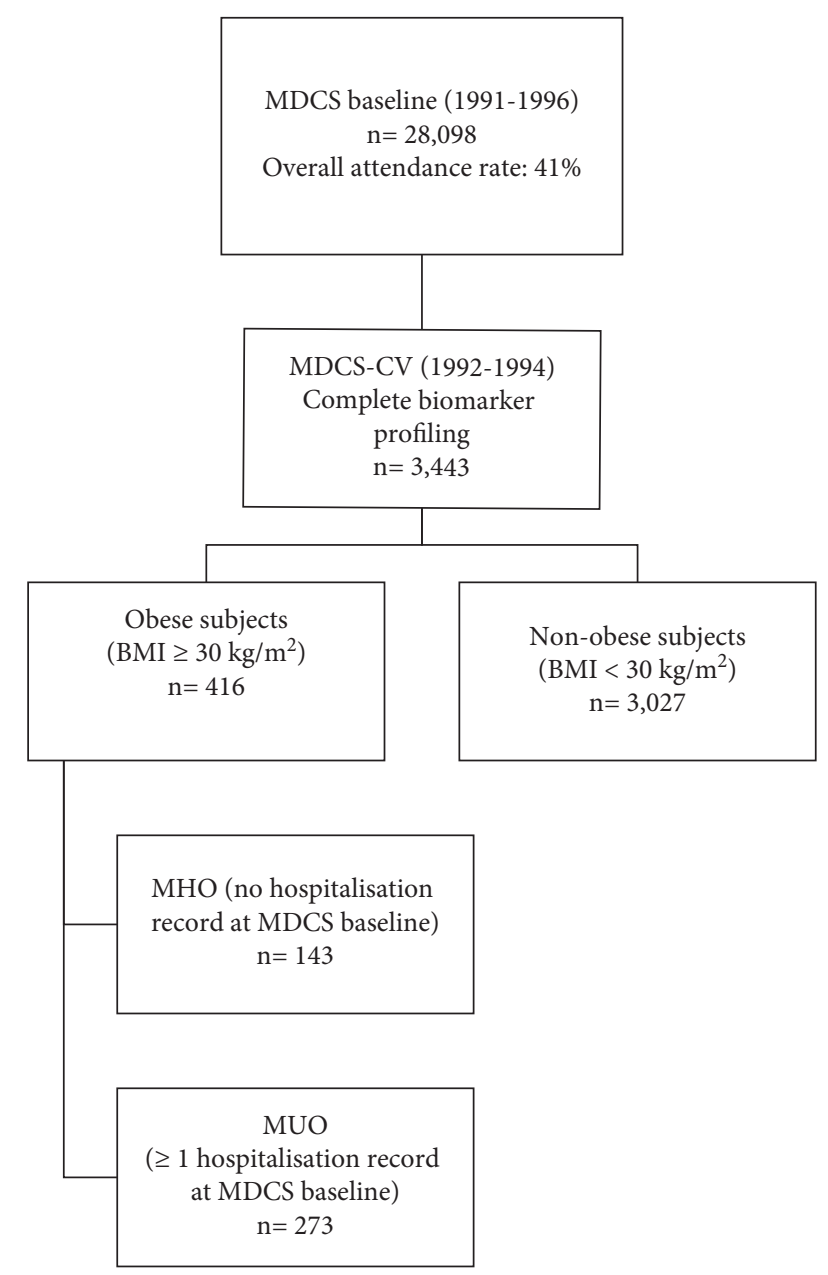

FIGURE 1: Flowchart of the MDCS-CV subcohort stratified for obese and nonobese subjects, respectively.

2.4. Protein Profiling. Fasting plasma levels of 136 proteins were measured using Olink Proseek Multiplex proximity extension assay (PEA) at the Clinical Biomarkers Facility, Science for Life Laboratory, Uppsala, Sweden. PEA uses two oligonucleotide-labelled antibodies per protein, which form a PCR reporter sequence when both antibodies are bound to the target protein. The reported sequence is quantified by real-time quantitative polymerase chain reaction [27].

2.5. Statistical Analysis. Prior to statistical analysis, missing values for all biomarkers (maximum 20\% missing allowed) were imputed using the NIPALS algorithm and were subsequently mean-centered and unit-variance scaled. Unsupervised dimension-reduction of each set of biomarker layers (metabolites, lipids, and proteins) was performed using principal component analysis (PCA). PCA was first performed for each biomarker layer in all obese participants and subsequently in all non-MUO participants in the same manner. For each biomarker layer, five principal components (PC) were calculated. In the participants with obesity, logistic regression models, adjusted for age and sex, were used to find associations between PCs and MHO (compared to MUO). PCs that were significantly associated with $\mathrm{MHO}$ were investigated for correlations with cardiometabolic risk factors using partial Spearman's correlation tests, adjusted for age and sex. Subsequent analysis in all non-MUO participants used logistic regression models to find associations between PCs and MHO (compared to NOC). All statistical analyses were performed in R 3.6.1. PCA and imputation were performed in the mixOmics [28] package and the partial Spearman's correlation tests in the ppcor [29] package. A $p$ value $<0.05$ was considered significant.

\section{Results}

We observed differences in several cardiometabolic risk factors between subjects with $\mathrm{MHO}$ subjects compared to MUO (Table 1). MHO participants had a more favorable cardiometabolic risk factor profile compared to MUO, including lower BMI and waist circumference, proportion of prescribed antihypertensive drugs, and fasting levels of glucose and triglycerides, as well as higher levels of $\mathrm{HDL}$ cholesterol. Apart from lower BMI, NOC participants were characterized by lower waist circumference, systolic and diastolic blood pressure $\mathrm{HbA}_{1 \mathrm{c}}$, proportion of antihypertensive drugs, and fasting levels of glucose, triglycerides, and LDL cholesterol, but higher levels of HDL cholesterol.

Biomarker profiles were constructed in participants with obesity $\left(\mathrm{BMI}>30 \mathrm{~kg} / \mathrm{m}^{2}\right)$, using PCA of three different biomarker layers, including either 112 metabolites, 184 lipids, or 136 proteins. The first five principal components (PC) in each biomarker layer could explain $41.8 \%$ of the metabolite variation, $63.8 \%$ of the lipid variation, and $53.1 \%$ of the protein variation, respectively (Table S2). To investigate whether the obesity biomarker patterns were related to $\mathrm{MHO}$, all 15 biomarker PCs were analyzed using logistic regression models. The second lipid PC (PL2) (odds ratio, OR 1.06, $p=0.018$ ) and the fifth protein PC (PP5) (OR 0.85, $p=0.013$ ) were associated with MHO (Figure 2). PL2 was dominated by positive contributions from phospholipids, such as sphingomyelins and phosphatidylcholine ethers, and negative contributions from triacylglycerides (Figure 3 ). The strongest positive contribution to PP5 was interleukin-1 receptor antagonist (IL1-RA) followed by leptin and fatty acid-binding protein 4 , while the strongest negative contributions were from galanin (Figure 4). Loadings for all PCs are presented in Tables S3-S5.

Both MHO-associated PCs were correlated with traditional cardiometabolic risk factors (Figure 5, Table S6). PL2 showed strong inverse correlations with plasma triglycerides (rho $=-0.67, p<0.001)$, HOMA-IR (rho $=-0.36, p<0.001$ ), and glucose (rho $=-0.32, p<0.001)$ and strong positive correlations with HDL cholesterol ( $\mathrm{rho}=0.59, p<0.001)$. PP5 was strongly correlated with CRP $($ rho $=0.36, p<0.001)$ and waist circumference (rho $=0.26, p<0.001$ ) but inversely correlated with HDL cholesterol (rho $=-0.27, p<0.001)$. All correlations between MHO-related PC and cardiometabolic risk factors are depicted in Figure 5.

When adjusting for combined components of the MetS, according to National Cholesterol Education Program panel III (NCEP III) criteria [30] (systolic blood pressure, plasma glucose, HDL cholesterol, triglycerides, and waist 
TABLE 1: Descriptive comparison and significance testing for MHO $(n=143)$ compared to MUO subjects $(n=273)$ and MHO compared to NOC subjects $(n=3,027)$, with standard deviation (SD) or percentage $(\%)$ for metric and categorical variables, respectively.

\begin{tabular}{|c|c|c|c|c|c|}
\hline Variable & MHO $(N=143)$ & MUO $(N=273)$ & $p$ & $\mathrm{NC}(N=3027)$ & $p$ \\
\hline Age (years) & $57.7(5.7)$ & $59.1(5.9)$ & 0.02 & $57.4(6.0)$ & 0.50 \\
\hline Sex (\% women) & 65.7 & 65.6 & 0.97 & 59.2 & 0.11 \\
\hline BMI $\left(\mathrm{kg} / \mathrm{m}^{2}\right)$ & $32.2(2.3)$ & $33.4(3.34)$ & $<0.001$ & $24.6(2.8)$ & $<0.001$ \\
\hline Waist $(\mathrm{cm})$ & $97.0(12)$ & $99.5(12)$ & 0.04 & $80.9(11)$ & $<0.001$ \\
\hline SBP (mmHg) & $150(19)$ & $148(19)$ & 0.51 & $140(19)$ & $<0.001$ \\
\hline DBP (mmHg) & $90.9(9.7)$ & $91.0(9.4)$ & 0.90 & $86.1(9.2)$ & $<0.001$ \\
\hline Smoker (\%) & 21.1 & 15.8 & 0.19 & 27.6 & 0.07 \\
\hline AHT drug (\%) & 19.6 & 37.7 & $<0.001$ & 14.1 & 0.11 \\
\hline Glucose $(\mathrm{mmol} / \mathrm{L})$ & $5.53(1.4)$ & $5.90(1.9)$ & 0.03 & $5.09(1.2)$ & $<0.001$ \\
\hline $\mathrm{HbA}_{1 \mathrm{c}}(\%)$ & $5.11(0.82)$ & $5.29(1.0)$ & 0.054 & $4.88(0.68)$ & 0.001 \\
\hline HOMA-IR & $3.66(7.5)$ & $3.81(4.0)$ & 0.82 & $1.67(1.9)$ & 0.002 \\
\hline $\mathrm{TG}(\mathrm{mmol} / \mathrm{L})$ & $1.57(0.72)$ & $1.75(0.80)$ & 0.02 & $1.25(0.60)$ & $<0.001$ \\
\hline HDL-C $(\mathrm{mmol} / \mathrm{L})$ & $1.27(0.32)$ & $1.20(0.28)$ & 0.03 & $1.43(0.38)$ & $<0.001$ \\
\hline LDL-C (mmol/L) & $4.35(1.1)$ & $4.35(1.1)$ & 0.99 & $4.13(0.97)$ & 0.02 \\
\hline CRP (mg/L) & $0.39(0.50)$ & $0.41(0.44)$ & 0.74 & $0.23(0.40)$ & $<0.001$ \\
\hline MetS (\%) & 49.7 & 61.5 & 0.03 & 13.6 & $<0.001$ \\
\hline
\end{tabular}

AHT: antihypertensive treatment; DBP: diastolic blood pressure; SBP: systolic blood pressure: HbAlc: glycated haemoglobin; HOMA-IR: homeostatic model assessment for insulin resistance; CRP: C-reactive protein; MetS: metabolic syndrome.

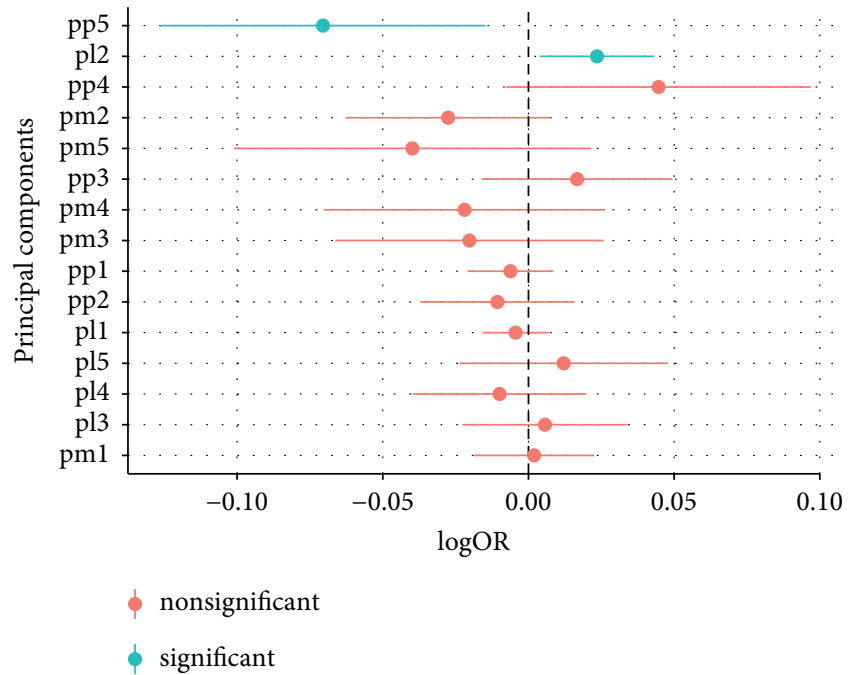

FIGURE 2: Logistic regression models, with significance testing, of the main PCs when comparing MHO (a) with MUO (b) subjects.

circumference), no significant differences could be seen between the PCs. Strong contributors to differences in PL2 were HDL cholesterol and triacylglycerides (Table 2).

PCA of three different biomarker layers was used to describe the biomarker variation of study participants without MUO. The first five principal components (PC) in each biomarker layer could explain $43.0 \%$ of the metabolite variation, $53.5 \%$ of the lipid variation, and $61.4 \%$ of the protein variation (Table S2). In general, there were larger differences in the biomarker pattern between NOC and MHO subjects, than between $\mathrm{MHO}$ and MUO subjects. Seven PCs, three protein PCs, two lipid PCs, and two metabolite PCs were associated with increased odds of $\mathrm{MHO}$ as compared to NOC (Figure 6). Similar to PP4 in the obese individuals, PP5, which was the PC that was most strongly associated with increased odds of MHO over NOC, had

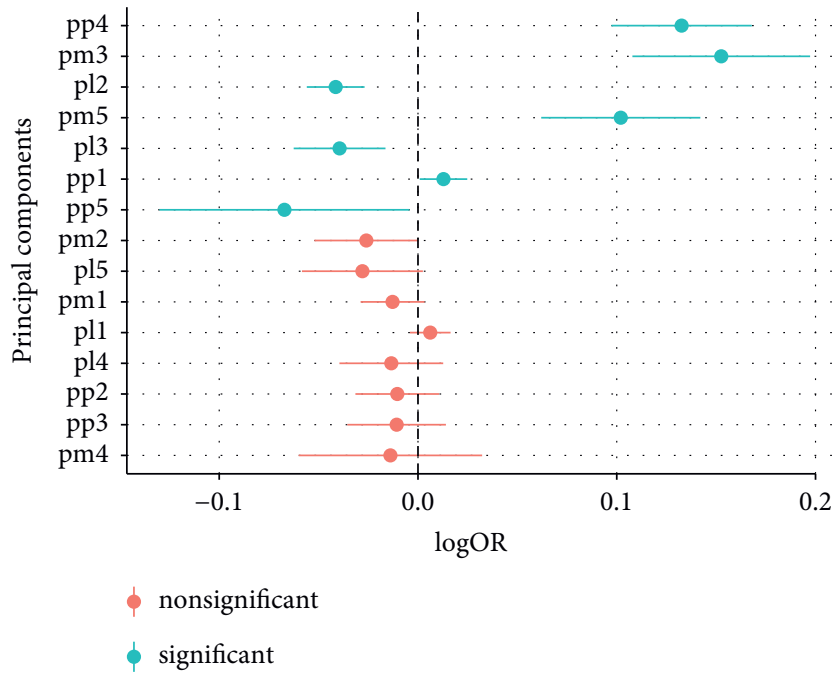

FIGURE 3: Main loadings for PL2, when comparing MHO with MUO.

strong positive contributions from IL1-RA and IL6, but negative contributions from galanin and pappalysin- 1 . The lipid PC, showing the largest differences between MHO and NOC, PL2, was dominated by negative contributions from triacylglycerides and positive contributions from phosphatidylcholine ethers, similar to PL2 in the obese population (Tables S7-S9).

\section{Discussion}

This observational study from an urban population ran an extensive biomarker profiling of 432 lipids, metabolites, and proteins across two distinct obese subgroups, $\mathrm{MHO}$ and MUO-an unexplored field as of yet. Key biomarker pattern findings include additional evidence of $\mathrm{MHO}$ individuals holding a more metabolically favorable lipid and glucose profile, that is, lower levels of traditional blood glucose and 


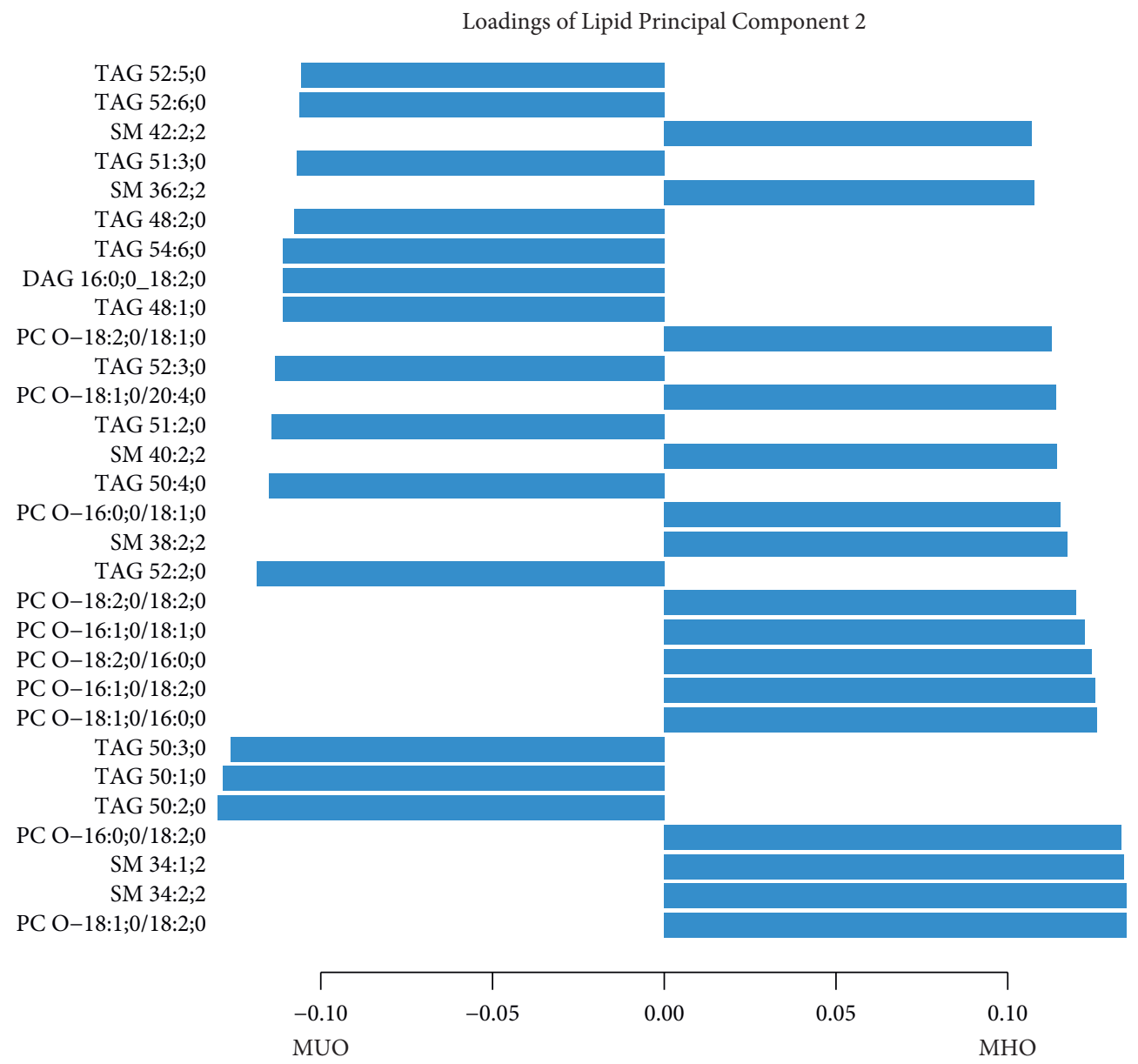

FIGURE 4: Main loadings for PP5, when comparing MHO with MUO.

triglycerides, as well as a trend of lower levels of metabolically unfavorable lipid biomarkers. Even if significance levels were modest, PCA discovered nominally significant proteomic and lipidomic biomarkers that differed between the MHO and MUO subgroups. These differences were to a large extent explained by factors related to the MetS. When comparing $\mathrm{MHO}$ individuals to NOC, PCA of selected biomarkers and descriptive data demonstrated expected findings of obesity-related parameters.

4.1. Lipidomics. Lipidomic patterns display MHO-related principal component (PL2) with negative contributions of triacylglycerides and diacylglycerides when compared to MUO. This supports the notion of a more benign lipid profile of $\mathrm{MHO}$ subjects, since higher levels of triglycerides mirror a more metabolically active adipose tissue as well as atherogenic properties, thus with the MetS [31]. Furthermore, glycerphospholipids (exclusively ether phosphatidylcholine) and sphingomyelins seem to be associated positively with the MHO-related PL2. It is unclear whether phospholipids contribute positively or negatively to cardiovascular disease and metabolic disorders [32, 33]. However, research shows that ether phosphatidylcholine with shorter fatty acids and smaller amounts of total double bonds (more positively correlated with $\mathrm{MHO}$ ) is increased in long-lived humans [34] - suggesting that perhaps $\mathrm{MHO}$ individuals deal better with oxygen stress than their counterparts, a theory supported by a previously cited systematic review, revealing that plasmalogens present a negative correlation with obesity, DM2, prediabetes, and CVD-all conditions associated with elevated levels of oxidative stress [32]. Moreover, sphingolipids (mainly sphingomyelin) seem to contribute to adipose tissue inflammation and the accompanying liver steatosis and insulin resistance; that is why their positive relationship with $\mathrm{MHO}$ appears more sophisticated than expected [32, 35]. The finding should be interpreted with caution given the relatively modest level of significance and number of PCs analyzed.

\subsection{Proteomics}

4.2.1. Interleukin-1 Receptor Antagonist (IL-1ra). Our study presented positive contributions of IL-1ra with the MUOrelated PP5, compared to $\mathrm{MHO}$. It has been debated whether IL-1ra is benign or if elevated levels of this biomarker present with adverse effects [36]. Indeed, it works as an inhibitor of the well-known proinflammatory cytokine interleukin-1 $\beta$ (IL-1 $\beta$ ), involved in the development of various chronic inflammatory disorders, as well as CVD and DM2 [37]. The randomized double-blind CANTOS study 
Loadings of Protein Principal Component 5

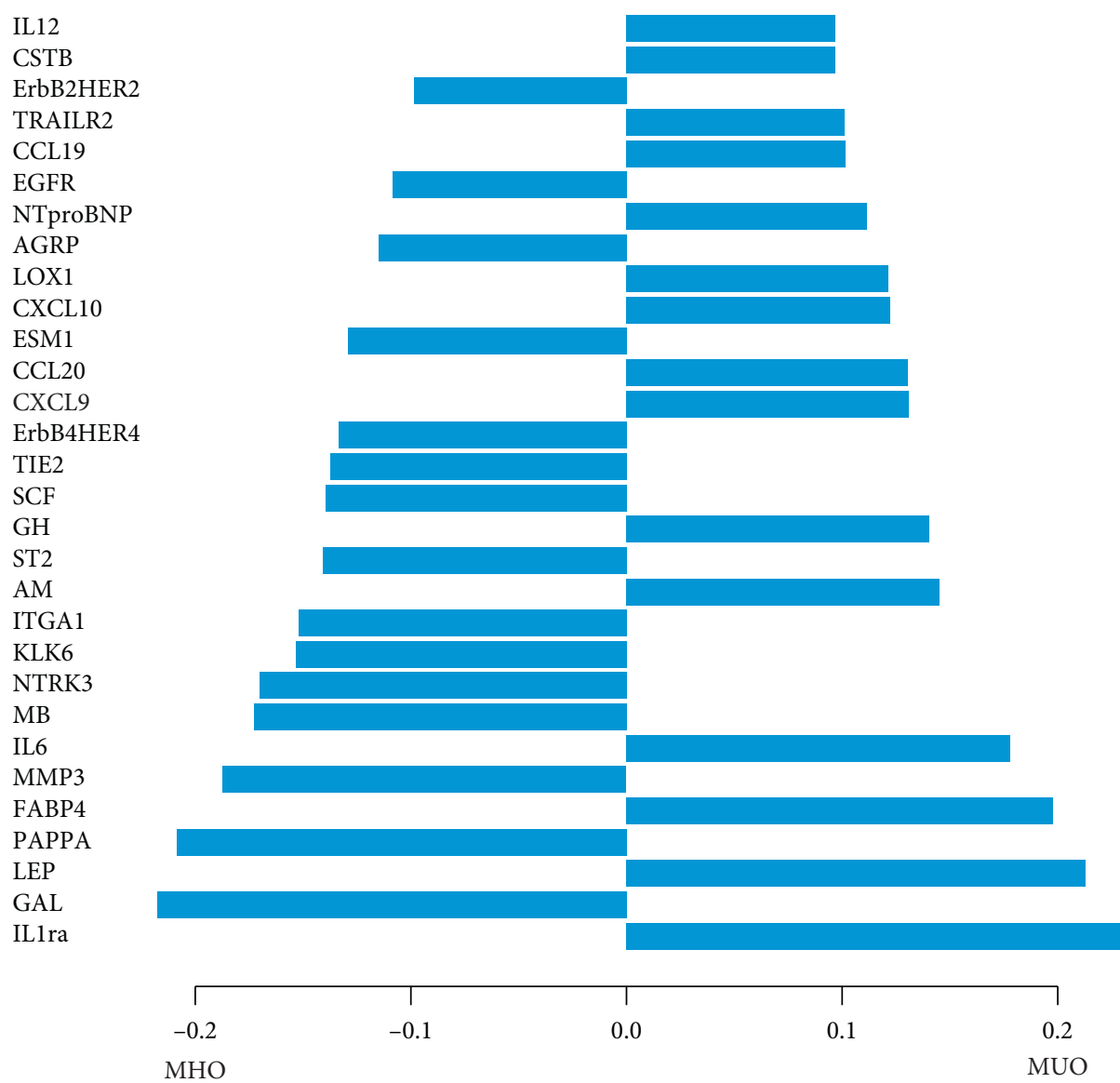

FIGURE 5: Correlation between biomarker principal components and cardiometabolic risk factors. Correlations between cardiometabolic risk factors and lipid principal component 2 (pl2) and protein principal component 5 (pp5) are expressed as partial Spearman's correlation coefficients, adjusted for age and sex. AHT: antihypertensive treatment; CRP: C-reactive protein; DBP: diastolic blood pressure; SBP: systolic blood pressure; HbA1c: glycated haemoglobin; HOMA-IR: homeostatic model assessment for insulin resistance.

contributed to the increasing evidence of positive effects of IL-1ra focused treatment, displaying that anti-inflammatory targeting with monoclonal antibodies against IL- $1 \beta$ significantly reduced recurrent cardiovascular events compared to placebo in postmyocardial infarction survivors [38]. However, conflicting data postulates that this protein is upregulated as a protective response to increased activities of IL$1 \beta$ and interleukin- $1 \alpha$ (IL- $1 \alpha$ ) [39]. Furthermore, additional findings hypothesize that IL-1RA might have harmful cardiovascular effects of its own and additionally prevent potentially positive effects of IL- $1 \alpha$ and IL- $1 \beta$ [36].

4.2.2. Galanin. An obesity-related neuropeptide was found negatively related to the MUO-correlated PP5, suggesting a relationship with MHO. Galanin is mainly involved in energy homeostasis, where increased hormone levels contribute to the development of obesity, through orexigenic effects, and also obesity-associated metabolic impairments, regardless of feeding regulation $[40,41]$. Nevertheless, one study reports potential positive effects of this hormone, where it seems to improve glucose metabolism and uptake, thus decreasing insulin resistance [42].
4.2.3. Leptin. Being a well-known biomarker for obesity, leptin correlated positively with MUO-related PP5. This adipocyte-derived hormone, increases with BMI and adipose tissue mass, suggesting that obese individuals develop an insensitivity to this hormone with increasing weight [43]. The hormone regulates the energy balance by inhibiting hunger mediated through the hypothalamus; hence, it works to reduce caloric intake and increase energy expenditure [44], suggesting that such obesity-promoting mechanisms might be more pronounced in MUO than in MHO.

4.3. Study Limitations. This is the first study of its kind, including 432 metabolites and proteins aiming to describe their relationship with metabolically healthy versus unhealthy obesity. Still, several limitations should be considered in this study. MDCS, although being a wellcharacterized, population-based prospective cohort with a large number of included individuals, had a relatively poor overall attendance rate $(41 \%)$ which could imply a health selection bias. Furthermore, both at baseline examination and reflected in our study sample, there exists a gender imbalance with a predominance of women. 
TABLE 2: Multiple regression model (linear logistic regression) displaying odds ratios which indicate associations between biomarker principal components and MHO, compared to MUO.

\begin{tabular}{lcccc}
\hline \multirow{2}{*}{ Model } & \multicolumn{2}{c}{ PP5 } & \multicolumn{2}{c}{ PL2 } \\
& $p$ & OR & $p$ & OR \\
\hline Model 1 (age + sex) & $0.01^{*}$ & 0.85 & $0.02^{*}$ & 1.06 \\
Model 1 + systolic blood pressure & $0.01^{*}$ & 0.85 & $0.02^{*}$ & 1.06 \\
Model 1 + plasma glucose & $0.02^{*}$ & 0.86 & 0.06 & 1.05 \\
Model 1 + HDL cholesterol & 0.06 & 0.88 & 0.35 & 1.03 \\
Model 1 + triglycerides & 0.04 & 0.87 & 0.24 & 1.04 \\
Model 1 + waist circumference & 0.07 & 0.91 & 0.08 & 1.04 \\
Model 1 + metabolic syndrome & 0.17 & 0.91 & 0.91 & 1.00 \\
\hline
\end{tabular}

Odds ratios (OR) indicate associations between biomarker principal components and $\mathrm{MHO}(=1)$, compared to $\mathrm{MUO}(=0)$. Model 1 was adjusted for age and sex. PL2: lipidomic principal component 2. PP5: proteomic principal component 5 . The model adjusted for metabolic syndrome was adjusted for all factors of the metabolic syndrome according to National Cholesterol Education Program panel III (NCEP III) criteria [30] (systolic blood pressure, fasting plasma glucose, HDL cholesterol, triglycerides, and waist circumference). $*$ Significant at $p<0.05$.

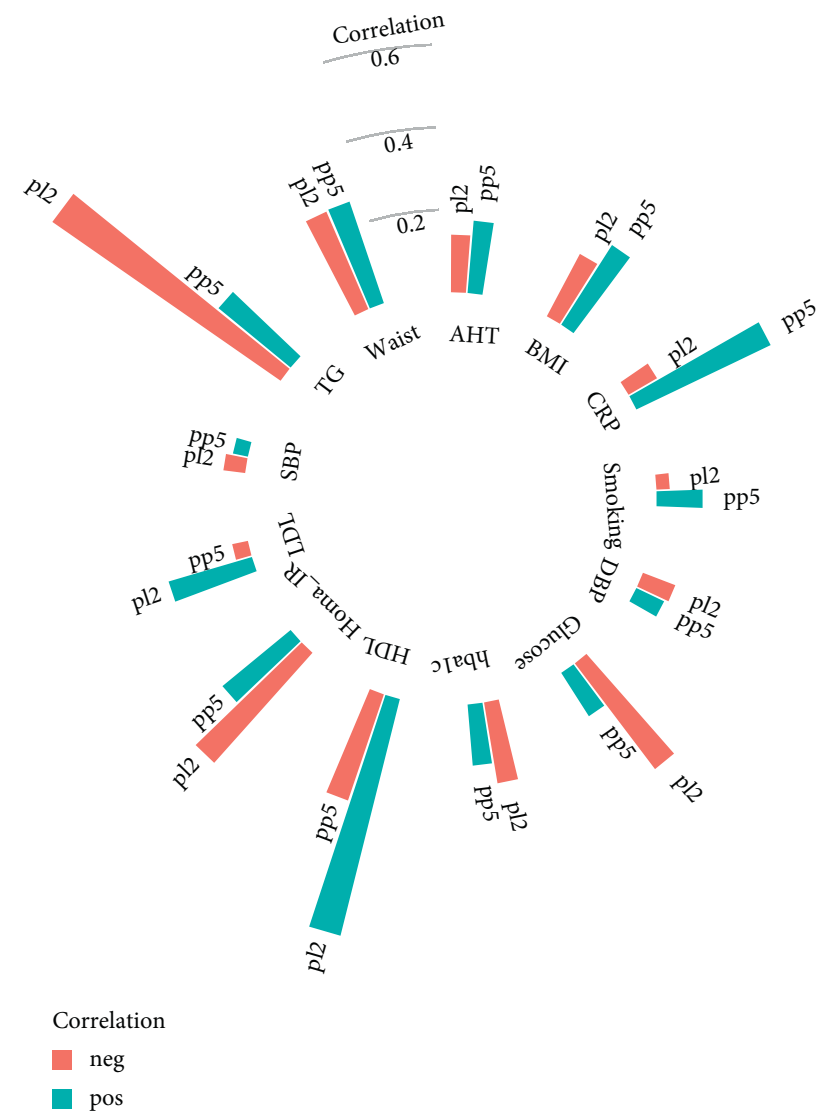

FIGURE 6: Logistic regression models, with significance testing, of the main PCs when comparing MHO (a) with NOC subjects (b).

A major limitation is the small sample size, resulting in limited power. Moreover, although we applied a data-reducing strategy, several derived PCs were tested with obesity phenotypes with the risk of false-positive results. This underlines the need for replication of the reported findings.
When performing a multiple regression analysis, adjusting for the components of the MetS [30], the associations of the biomarker PCs when comparing $\mathrm{MHO}$ and MUO individuals were attenuated (Table 2). This suggests that the difference of biomarker variation between $\mathrm{MHO}$ and MUO subjects in part could be explained by the MetS. Thus, one might argue that to keep the MHO phenotypic state and avoid hospitalization, there should be an ambition of the individual for weight stability and keeping a healthy lifestyle to avoid transformation into MUO linked to the MetS.

\section{Conclusion}

We have performed a plasma metabolic and protein profiling of $\mathrm{MHO}$ and MUO individuals, defined by absence (MHO) or presence (MUO) of a history of hospitalization for a somatic disease until midlife. Despite relatively weak associations, this novel approach confirms that $\mathrm{MHO}$ individuals present with a positive association with phosphatidylcholine ethers and sphingomyelins, as well as negative associations with triacyl- and diacylglycerides compared to MUO subjects. Furthermore, MHO individuals are characterized by the downregulation of potentially harmful proteomic biomarkers, compared to their MUO counterparts. A large part of the difference could be explained by the influence of MetS. Our research is in line with previous findings, although a unique and extensive biomarker profiling presents novel data on potential differentiating traits between these two obese phenotypes.

$\begin{array}{ll}\text { Abbreviations } \\ \text { CVD: } & \text { Cardiovascular disease } \\ \text { CRP: } & \text { C-reactive protein } \\ \text { DM2: } & \text { Type } 2 \text { diabetes } \\ \text { FABP4: } & \text { Fatty acid-binding protein } 4 \\ \text { HOMA- } & \text { Homeostatic model assessment for insulin } \\ \text { IR: } & \text { resistance } \\ \text { IL-1RA: } & \text { Interleukin-1 receptor antagonist } \\ \text { MDCS: } & \text { Malmö diet cancer study } \\ \text { MDCS- } & \text { Malmö diet cancer study-cardiovascular arm } \\ \text { CV: } & \\ \text { MHO: } & \text { Metabolically Healthy Obesity } \\ \text { MUO: } & \text { Metabolically unhealthy obesity } \\ \text { NAFLD: } & \text { Nonalcoholic fatty liver disease } \\ \text { NOC: } & \text { Nonobese controls } \\ \text { PAPPA: } & \text { Pappalysin-1 } \\ \text { PC: } & \text { Principal component } \\ \text { PCA: } & \text { Principal component analysis. }\end{array}$

\section{Data Availability}

All requests for data access should be addressed to the corresponding author. Proposals requesting data access will have to specify how they plan to use the data. 


\section{Conflicts of Interest}

JK, PMN, OM, GE, MM, and FO report no conflicts of interest. MJG is an employee of Lipotype GmbH. EB is an employee of AstraZeneca.

\section{Acknowledgments}

The study was funded by the Research Council of Sweden, Research Funds of Region Skåne, and the Skane University Hospital Funds and Foundations (Sweden), Knut and Alice Wallenberg Foundation, the Göran Gustafsson Foundation, the Swedish Heart and Lung Foundation, the Swedish Research Council, the Novo Nordisk Foundation, the European Research Council (ERC-ADG-885003), Swedish Foundation for Strategic Research for IRC15-0067, the Wallenberg Centre for Molecular Medicine, Lund University, and the Ernhold Lundström Foundation. This study was part of the AIR Lund (Artificially Intelligent use of Registers at Lund University) research environment and received funding from the Swedish Research Council (VR; Grant no. 2019-00198). The Knut och Alice Wallenbergs Stiftelse is acknowledged for generous support.

\section{Supplementary Materials}

Supplementary tables (S1-S9): index and contain additional data as follows: S1: table of SwissLipids IDs. S2: proportion of explained variance for the first 5 PCs in each biomarker layer (metabolite, lipid, and protein). The proportion of explained variance is presented for the principal component analysis performed for the obese participants $(n=416)$ $(\mathrm{MHO}+\mathrm{MUO})$ and the non-MUO (MUO + NOC) $(n=3,027)$. S3: loadings of metabolite PCs $1-5$ (MHO vs. MUO). S4: loadings of lipid PCs 1-5 (MHO vs. MUO). S5: loadings of protein PCs 1-5 (MHO vs. MUO). S6: correlation between biomarker PCs (PL2, PP5) and cardiometabolic risk factors. S7: loadings of metabolite PCs 1-5 (MHO vs. NOC). S8: loadings of lipid PCs 1-5 (MHO vs. NOC). S9: loadings of protein PCs 1-5 (MHO vs. NOC). (Supplementary Materials)

\section{References}

[1] H. B. Hubert, M. Feinleib, P. M. McNamara, and W. P. Castelli, "Obesity as an independent risk factor for cardiovascular disease: a 26 year follow-up of participants in the framingham heart study," Circulation, vol. 67, no. 5, pp. 968-977, 1983.

[2] G. I. Smith, B. Mittendorfer, and S. Klein, "Metabolically healthy obesity: facts and fantasies," Journal of Clinical Investigation, vol. 129, no. 10, pp. 3978-3989, 2019.

[3] C. Iacobini, G. Pugliese, C. Blasetti Fantauzzi, M. Federici, and S. Menini, "Metabolically healthy versus metabolically unhealthy obesity," Metabolism, vol. 92, pp. 51-60, 2019.

[4] P. M. Nilsson, J. Korduner, and M. Magnusson, "Metabolically healthy obesity (MHO)-new research directions for personalised medicine in cardiovascular prevention," Current Hypertension Reports, vol. 22, no. 2, p. 18, 2020.

[5] N. Eckel, Y. Li, O. Kuxhaus, N. Stefan, F. B. Hu, and M. B. Schulze, "Transition from metabolic healthy to unhealthy phenotypes and association with cardiovascular disease risk across BMI categories in 90257 women (the nurses' health study): 30 year follow-up from a prospective cohort study," The Lancet Diabetes \& Endocrinology, vol. 6, no. 9, pp. 714-724, 2018.

[6] J. B. Echouffo-Tcheugui, M. I. Short, V. Xanthakis et al., "Natural history of obesity subphenotypes: dynamic changes over two decades and prognosis in the framingham heart study," The Journal of Clinical Endocrinology \& Metabolism, vol. 104, no. 3, pp. 738-752, 2019.

[7] A. Munoz-Garach, I. Cornejo-Pareja, and F. J. Tinahones, "Does metabolically healthy obesity exist?" Nutrients, vol. 8, no. 6, 2016.

[8] C. K. Kramer, B. Zinman, and R. Retnakaran, "Are metabolically healthy overweight and obesity benign conditions?: a systematic review and meta-analysis," Annals of Internal Medicine, vol. 159, no. 11, pp. 758-769, 2013.

[9] J. Korduner, E. Bachus, A. Jujic, M. Magnusson, and P. M. Nilsson, "Metabolically healthy obesity (MHO) in the Malmo diet cancer study-epidemiology and prospective risks," Obesity Research and Clinical Practice, vol. 13, 2019.

[10] R. Zheng, D. Zhou, and Y. Zhu, "The long-term prognosis of cardiovascular disease and all-cause mortality for metabolically healthy obesity: a systematic review and meta-analysis," Journal of Epidemiology and Community Health, vol. 70, no. 10 , pp. 1024-1031, 2016.

[11] T. L. Yeh, H. H. Chen, S. Y. Tsai, C. Y. Lin, S. J. Liu, and K. L. Chien, "The relationship between metabolically healthy obesity and the risk of cardiovascular disease: a systematic review and meta-analysis," Journal of Clinical Medicine, vol. 8, no. $8,2019$.

[12] F. Ottosson, L. Brunkwall, U. Ericson et al., "Connection between bmi-related plasma metabolite profile and gut microbiota," The Journal of Clinical Endocrinology \& Metabolism, vol. 103, no. 4, pp. 1491-1501, 2018.

[13] M. E. Piché, P. Poirier, I. Lemieux, and J. P. Després, "Overview of epidemiology and contribution of obesity and body fat distribution to cardiovascular disease: an update," Progress in Cardiovascular Diseases, vol. 61, no. 2, pp. 103-113, 2018.

[14] G. H. Goossens, "The metabolic phenotype in obesity: fat mass, body fat distribution, and adipose tissue function," Obesity Facts, vol. 10, no. 3, pp. 207-215, 2017.

[15] N. Babic, A. Valjevac, A. Zaciragic, N. Avdagic, S. Zukic, and S. Hasic, "The triglyceride/HDL ratio and triglyceride glucose index as predictors of glycemic control in patients with diabetes mellitus type 2," Medical Archives, vol. 73, no. 3, pp. 163-168, 2019.

[16] S. H. Lee, K. Han, H. K. Yang et al., "Identifying subgroups of obesity using the product of triglycerides and glucose: the Korea national health and nutrition examination survey, 2008-2010," Clinical Endocrinology, vol. 82, no. 2, pp. 213-220, 2015

[17] A. E. Achari and S. K. Jain, "Adiponectin, a therapeutic target for obesity, diabetes, and endothelial dysfunction," International Journal of Molecular Sciences, vol. 18, no. 6, 2017.

[18] J. Li, J. Song, Y. Y. Zaytseva et al., "An obligatory role for neurotensin in high-fat-diet-induced obesity," Nature, vol. 533, no. 7603, pp. 411-415, 2016.

[19] J. Manjer, S. Carlsson, S. Elmståhl et al., “The Malmo diet and cancer study: representativity, cancer incidence and mortality in participants and non-participants," European Journal of Cancer Prevention, vol. 10, no. 6, pp. 489-499, 2001. 
[20] G. Berglund, S. Elmståhl, L. Janzon, and S. A. Larsson, "The Malmo diet and cancer study. design and feasibility," Journal of Internal Medicine, vol. 233, no. 1, pp. 45-51, 1993.

[21] M. Rosvall, L. Janzon, G. Berglund, G. Engström, and B. Hedblad, "Incidence of stroke is related to carotid IMT even in the absence of plaque," Atherosclerosis, vol. 179, no. 2, pp. 325-331, 2005.

[22] M. Rosvall, M. Persson, G. Östling et al., "Risk factors for the progression of carotid intima-media thickness over a 16 year follow-up period: the Malmo diet and cancer study," Atherosclerosis, vol. 239, no. 2, pp. 615-621, 2015.

[23] F. Ottosson, U. Ericson, P. Almgren et al., "Postprandial levels of branch chained and aromatic amino acids associate with fasting glycaemia," Journal of Amino Acids, vol. 2016, Article ID 8576730, 9 pages, 2016.

[24] E. Smith, F. Ottosson, S. Hellstrand et al., "Ergothioneine is associated with reduced mortality and decreased risk of cardiovascular disease," Heart, vol. 106, 2019.

[25] M. A. Surma, R. Herzog, A. Vasilj et al., "An automated shotgun lipidomics platform for high throughput, comprehensive, and quantitative analysis of blood plasma intact lipids," European Journal of Lipid Science and Technology, vol. 117, no. 10, pp. 1540-1549, 2015.

[26] L. Aimo, R. Liechti, N. Hyka-Nouspikel et al., "The SwissLipids knowledgebase for lipid biology," Bioinformatics, vol. 31, no. 17, pp. 2860-2866, 2015.

[27] E. Assarsson, M. Lundberg, G. Holmquist et al., "Homogenous 96-plex PEA immunoassay exhibiting high sensitivity, specificity, and excellent scalability," PLoS One, vol. 9, no. 4, Article ID e95192, 2014.

[28] F. Rohart, B. Gautier, A. Singh, and K. A. Lê Cao, "mixOmics: an R package for 'omics feature selection and multiple data integration," PLoS Computational Biology, vol. 13, no. 11, Article ID e1005752, 2017.

[29] S. Kim, "ppcor: an R package for a fast calculation to semipartial correlation coefficients," Communications for Statistical Applications and Methods, vol. 22, no. 6, pp. 665-674, 2015.

[30] National Cholesterol Education Program, "Expert panel on detection E, treatment of high blood cholesterol in a. third report of the national cholesterol education program (NCEP) expert panel on detection, evaluation, and treatment of high blood cholesterol in adults (adult treatment panel III) final report," Circulation, vol. 106, no. 25, pp. 3143-3421, 2002.

[31] S. M. Grundy, "Hypertriglyceridemia, atherogenic dyslipidemia, and the metabolic syndrome," The American Journal of Cardiology, vol. 81, no. 4A, pp. 18B-25B, 1998.

[32] P. J. Meikle and S. A. Summers, "Sphingolipids and phospholipids in insulin resistance and related metabolic disorders," Nature Reviews Endocrinology, vol. 13, no. 2, pp. 79-91, 2017.

[33] K. Petkevicius, S. Virtue, G. Bidault et al., “Accelerated phosphatidylcholine turnover in macrophages promotes adipose tissue inflammation in obesity," Elife, vol. 8, 2019.

[34] I. Pradas, M. Jové, K. Huynh et al., "Exceptional human longevity is associated with a specific plasma phenotype of ether lipids," Redox Biology, vol. 21, Article ID 101127, 2019.

[35] M. J. Gerl, C. Klose, M. A. Surma et al., "Machine learning of human plasma lipidomes for obesity estimation in a large population cohort," PLoS Biology, vol. 17, no. 10, Article ID e3000443, 2019.

[36] C. Herder and M. Y. Donath, "Interleukin-1 receptor antagonist: friend or foe to the heart?" The Lancet Diabetes \& Endocrinology, vol. 3, no. 4, pp. 228-229, 2015.
[37] W. F. Fearon and D. T. Fearon, "Inflammation and cardiovascular disease: role of the interleukin-1 receptor antagonist," Circulation, vol. 117, no. 20, pp. 2577-2579, 2008.

[38] P. M. Ridker, B. M. Everett, T. Thuren et al., "Antiinflammatory therapy with canakinumab for atherosclerotic disease," New England Journal of Medicine, vol. 377, no. 12, pp. 1119-1131, 2017.

[39] C. Herder, M. Carstensen, and D. M. Ouwens, "Anti-inflammatory cytokines and risk of type 2 diabetes," Diabetes Obesity and Metabolism, vol. 15, no. Suppl 3, pp. 39-50, 2013.

[40] B. Baranowska, M. Radzikowska, E. Wasilewska-Dziubinska, K. Roguski, and M. Borowiec, "Disturbed release of gastrointestinal peptides in anorexia nervosa and in obesity," $D i$ abetes, Obesity and Metabolism, vol. 2, no. 2, pp. 99-103, 2000.

[41] N. J. Poritsanos, T. M. Mizuno, M. E. Lautatzis, and M. Vrontakis, "Chronic increase of circulating galanin levels induces obesity and marked alterations in lipid metabolism similar to metabolic syndrome," International Journal of Obesity, vol. 33, no. 12, pp. 1381-1389, 2009.

[42] P. Fang, M. Yu, M. Shi, P. Bo, and Z. Zhang, "Galanin peptide family regulation of glucose metabolism," Frontiers in Neuroendocrinology, vol. 56, Article ID 100801, 2020.

[43] R. V. Considine, M. K. Sinha, M. L. Heiman et al., "Serum immunoreactive-leptin concentrations in normal-weight and obese humans," New England Journal of Medicine, vol. 334, no. 5, pp. 292-295, 1996.

[44] J. L. Halaas, C. Boozer, J. Blair-West, N. Fidahusein, D. A. Denton, and J. M. Friedman, "Physiological response to long-term peripheral and central leptin infusion in lean and obese mice," Proceedings of the National Academy of Sciences, vol. 94, no. 16, pp. 8878-8883, 1997. 